\title{
Differences of crystal structure and dynamics between a soft porous nanocrystal and a bulk crystal.
}

\section{$\operatorname{AUTHOR}(S):$}

Hijikata, Yuh; Horike, Satoshi; Tanaka, Daisuke; Groll, Juergen; Mizuno, Motohiro; Kim, Jungeun; Takata, Masaki; Kitagawa, Susumu

\section{CITATION:}

Hijikata, Yuh ...[et al]. Differences of crystal structure and dynamics between a soft porous nanocrystal and a bulk crystal.. Chemical communications 2011, 47(27): 7632-7634

\section{ISSUE DATE:}

2011-07-21

URL:

http://hdl.handle.net/2433/156800

\section{RIGHT:}

(C) Royal Society of Chemistry 2011.; This is not the published version. Please cite only the published version.; この論文は出版社版でありませ ん。引用の際には出版社版をご確認ご利用ください。 


\title{
Differences of crystal structure and dynamics between soft porous nanocrystal and bulk crystal
}

\author{
Yuh Hijikata, ${ }^{a}$ Satoshi Horike, ${ }^{a}$ Daisuke Tanaka, ${ }^{a, b}$ Juergen Groll, ${ }^{b}$ Motohiro Mizuno, ${ }^{c}$ Jungeun Kim, ${ }^{d}$ \\ Masaki Takata ${ }^{d, e}$ and Susumu Kitagawa*a,f,g
}

\author{
${ }_{5}$ Received (in $\left.X X X, X X X\right)$ Xth $X X X X X X X X X 20 X X$, Accepted Xth XXXXXXXXX 20XX \\ DOI: $10.1039 / b 000000 x$
}

We have demonstrated downsizing effects of the soft porous crystal, [Zn(isophthalate)(4,4'-bipyridyl) $]_{n}$ (CID-1) on the adsorption behavior between CID-1 and CID-1 nanocrystal

${ }_{10}$ (NCID-1). The difference results from the packing crystal structures and the dynamics of the frameworks.

Downsizing effects of condensed materials such as metals and metal oxides have attracted the interest of researchers because their electronic properties change depending on their particle 15 size. ${ }^{1}$ Downsized porous materials also have been applied for last decade to adsorption, separation, drug delivery, and heterogeneous catalysis. ${ }^{2}$ Recently, porous coordination polymers (PCPs) or metal-organic frameworks (MOFs) constructed from metal ions and organic linkers have emerged as new functional 20 crystalline porous materials because of the high architectural controllability of the structures. ${ }^{3}$ These materials can be classified as either rigid (robust) porous frameworks or flexible porous frameworks. ${ }^{4}$ The flexible frameworks show unconventional porous functions for gas separation and storage via a phase 25 transition, and unique functions originating from their flexibility have been reported. ${ }^{5}$ Although nanocrystals of PCPs have been synthesized intensively, ${ }^{6}$ effects on the downsizing of PCPs such as the structure of metal or metal oxide nanoparticles have not been investigated, especially for flexible PCPs. Downsizing 30 flexible PCP crystals may more greatly influence the adsorption properties than downsizing rigid type frameworks. ${ }^{7}$ Here we discovered marked differences of crystal structure and dynamic properties between the bulk and nanoscale crystals of a flexible PCP. These features closely correlate with adsorption properties.

35 Observations of differences of structure and dynamics between bulk and nanocrystals have not been reported so far and the robust or three-dimensional (3-D) PCP/MOF nanocrystals do not show these similar differences. ${ }^{6}$ We reported the synthesis of nanocrystals of the flexible coordination polymer, $[\mathrm{Zn} \text { (ip)(bpy) }]_{n}$ 40 (CID-1; CID = coordination polymer with interdigitated structure), which is constructed from two-dimensional (2-D) layers built with $\mathrm{Zn}^{2+}$, isophthalate (ip) and 4,4' -bipyridyl (bpy). ${ }^{7-}$ ${ }^{8}$ The crystals were synthesized by ultrasonication with AOT as an anionic surfactant which exists on only the surface of particles 45 such as other 3D frameworks with modified exterior surface. ${ }^{9}$ The main size of bulk CID-1 and CID-1 nanocrystals (NCID-1) is around $5 \mu \mathrm{m}$ wide $\times 20 \mu \mathrm{m}$ long and $50 \mathrm{~nm}$ wide $\times 320 \mathrm{~nm}$ long, respectively, with a distribution of particle $\operatorname{size}^{7}$ (ESI $\dagger$ ). The stepwise methanol gas adsorption isotherm of CID-1 changes to a 50 gradual, single profile in NCID-1, which indicates that the mechanism of gas adsorption and diffusion is significantly dependent on the particle size. However, the structure and dynamics have not been well characterized.

In this work, we investigated the packing structure of 2-D 55 layers using X-ray analysis because the initial structural phase dominates the adsorption process. The structure of CID-1 showed zero-dimensional (0-D) pores that require the reorientation of organic linkers in the framework for gas diffusion into pores. ${ }^{10}$ We also observed the dynamics using solid-state ${ }^{2} \mathrm{H}$ NMR.

60 The as-synthesized phases of CID-1 and NCID-1, both of which contain $N, N$-dimethylformamide (DMF) as guest molecules, show quite similar structures as supported by their $\mathrm{X}$ ray powder diffraction (XRD) patterns (ESI $\uparrow$ ). On the other hand, their guest-free phases have different X-ray patterns, especially ${ }_{65}$ the peaks at around $2 \theta=10^{\circ}$, as shown in Fig. 1 . That is, the initial structures of CID-1 and NCID-1 for gas adsorption are different. To obtain the structural information in detail, we determined the structure of guest-free CID-1 using single-crystal $\mathrm{X}$-ray analysis and obtained the cell parameters shown in Table 1 70 and Fig. 2 (ESI $\dagger$ ). The cell parameters of guest-free NCID-1 were also determined using LeBail analysis of powder diffraction obtained from synchrotron powder diffraction. The cell parameters of CID-1つDMF and NCID-1つDMF showed quite similar values $(\mathrm{ESI}+$ ). Compared with CID-1, the cell parameters 75 of NCID- 1 are $0.6 \AA$ longer in the $a$ axis and $0.4 \AA$ shorter in the
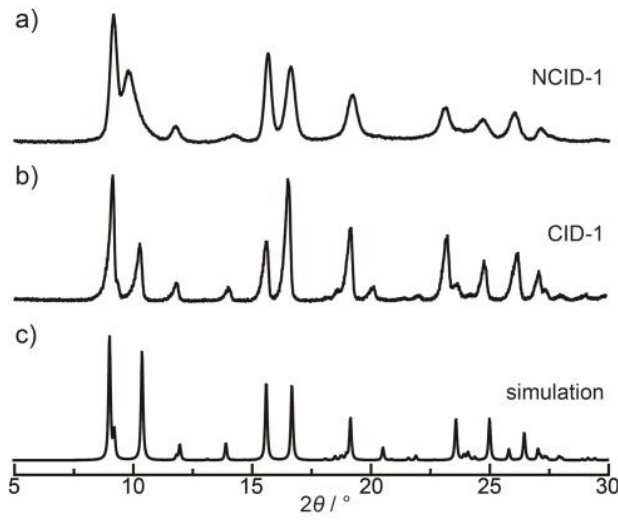

Fig. 1 XRD patterns of (a) NCID-1 and (b) CID-1, and (c) the simulated pattern of CID-1 produced using X-ray diffraction data from a single crystal. 
Table 1 Cell parameters of CID-1 and NCID-1

$\begin{array}{ccccccc} & a(\AA) & b(\AA) & c(\AA) & \alpha\left(^{\circ}\right) & \beta\left(^{\circ}\right) & \gamma\left({ }^{\circ}\right) \\ \text { CID-1 } & 8.95 & 10.08 & 10.12 & 78.20 & 73.26 & 79.15 \\ \text { NCID-1 } & 9.55 & 10.03 & 9.71 & 77.9 & 72.3 & 79.0 \\ \Delta^{a} & 0.60 & 0.05 & 0.41 & 0.30 & 0.96 & 0.15\end{array}$

${ }^{a}$ The values of $\Delta$ give the difference in cell parameters between guestfree CID-1 and NCID-1 frameworks.

$c$ axis, with little difference in the $b$ axis. The changes of the cell 5 parameters between the NCID- 1 and CID- 1 are $6.7 \%$ for the $a$ axis and $3.0 \%$ for the $c$ axis. The unit cell distortion accompanied by a downsizing effect is also observable in several metals and metal oxides. ${ }^{11}$ The changes of cell parameters mean that the packing structure of 2-D layers is different between CID-1 and ${ }_{10}$ NCID-1, although they have the same connectivity along the $b$ axis in 2-D layers. A schematic description of the structural differences is shown in Fig. 2. The changes are in the sliding of the 2-D layers. Considering that the largely distorted $a c$ plane is perpendicular to the 2-D layers of the structure, the differences in 15 cell parameters are derived from the structural freedom in the packing of the 2-D layers via van der Waals interactions. Such distortion of networks between bulk crystal and nanocrystal structure has not been observed in other PCPs having 3-D networks via coordination bonds, ${ }^{6 b-61}$ even if they have structural 20 flexibility. ${ }^{6 \mathrm{~m}}$ The observation of different XRD patterns between nano- and bulk crystals of the 2-D framework was shown, although explanations for the difference in the structures were not given. $^{12}$

The maximum cross sections of the pore entrance in CID-1 25 and CID-1 $\supset \mathrm{DMF}$ from the crystal structures are $1.8 \AA \times 4.0 \AA$ and $2.5 \AA \times 4.1 \AA$, respectively. On the other hand, the cross section of DMF that can be accommodated in the cavity of CID-1 is at least $3.2 \AA \times 5.0 \AA$. Comparison of these sizes indicates that CID-1 has 0-D pores before and after guest accommodation, and 30 a structural reorientation of organic ligands is required in the adsorption process, as a correlation of framework mobility and guest accommodation has been suggested. ${ }^{10,13}$ Then the mobility of bpy constructing the diffusion path for guest molecules was compared between CID-1 and NCID-1. To observe the

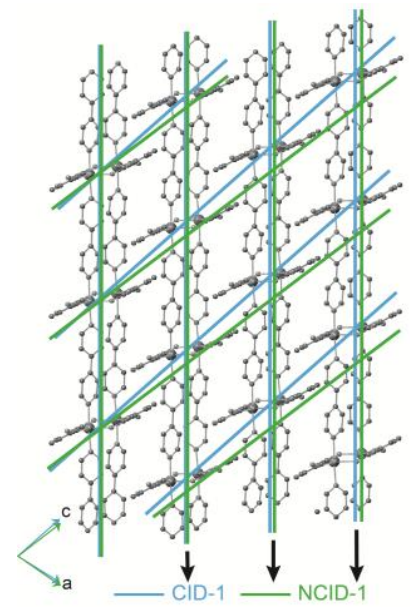

Fig. 2 Single crystal structure of guest-free CID-1 and schematic description of the changes of cell parameters between CID-1 and NCID-1, and the sliding of the 2-D layers. Blue and green grids represent the periodicity of the relative positions of isophthalate in the 2-D layers from 40 the direction of the $b$ axis in CID-1 and NCID-1, respectively.
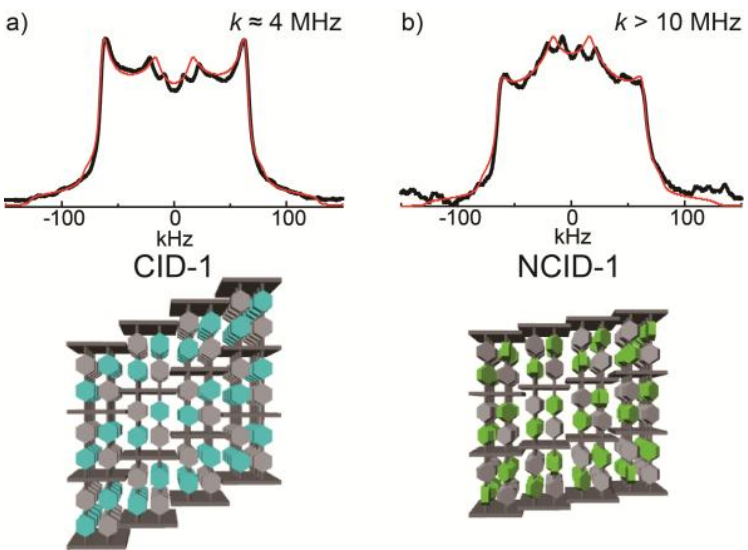

Fig. 3 The ${ }^{2} \mathrm{H}$ NMR spectra of (a) CID-1- $d$ and (b) NCID-1- $d$ at $298 \mathrm{~K}$ with schematic illustrations of the frameworks. $k$ is the motion rate constant. Black lines show experimental spectra and red lines are 45 simulated spectra. Blue and green hexagons of bpy have higher mobility than the gray parts of bpy.

framework dynamics using solid-state ${ }^{2} \mathrm{H}$ NMR, we synthesized CID-1- $d$ and NCID-1- $d$ from deuterated bpy ligand (bpy- $d_{8}$ ). ${ }^{14}$ Solid-state ${ }^{2} \mathrm{H}$ NMR measurements were carried out to obtain 50 local information regarding the molecular dynamics in combination with simulation for their spectra. Spectra of ${ }^{2} \mathrm{H}$ NMR for guest-free CID-1- $d$ and NCID-1- $d$ at 298 K are shown in Fig. 3. They have different line shapes, which indicates that the motion rate constant $k$ of bpy- $d_{8}$ in CID-1- $d$ and NCID-1- $d$ is 55 different. We simulated the observed spectra using a customwritten FORTRAN program to investigate the difference between them $($ ESI $\uparrow) .{ }^{15}$ These spectra were simulated as a two-site rotational model along the $C_{2}$ axis of bpy based on the singlecrystal structure of CID- 1 .

60 The crystal structure of CID-1 has two crystallographically independent pyridyl rings. NMR simulation suggested that one pyridyl ring of bpy has higher mobility, and the other has lower mobility, because lower-mobility pyridyl rings are restrained by the steric hindrance of isophthalate in the adjacent 2-D layer, 65 shown in Fig. 3a as gray hexagonal units. The pyridyl rings with higher mobility are shown in Fig. 3a as a blue hexagonal moiety. We simulated the observed spectra based on a single crystal of CID-1 and estimated that the rotational frequency of the higherand lower- mobility pyridyls are $c a$. $k=4 \mathrm{MHz}$ and less than $k=$ $701 \mathrm{kHz}$ (static state in NMR time scale) around the $C_{2}$ axis of bpy, respectively, because the observable range of frequency using ${ }^{2} \mathrm{H}$ NMR is from $10^{3}$ to $10^{7} \mathrm{~Hz} .{ }^{16}$ Meanwhile, the mobility of the bpy rings in NCID-1 is significantly accelerated, and the frequency of the pyridyl ring with higher mobility in NCID-1 reaches more 75 than $k=10 \mathrm{MHz}$ and is shown in Fig. $3 \mathrm{~b}$ as green hexagons. Additionally, the pyridyl rings with lower mobility also start to move ( $c a . k=8.0 \mathrm{kHz}$ ) in NCID-1. Although the observed small central peaks have not been reproduced, the change in the typical Pake patterns in CID-1 clearly indicate that an increase of 80 mobility in NCID-1 compared with that in CID-1 occurred. The changes in the relative position and interaction of 2-D layers loosen the restraint of bpy, resulting in its significantly enhanced mobility in NCID-1 compared with CID-1. The structural and dynamic changes in NCID-1 induce changes in adsorption ${ }_{85}$ behavior. The initial phase of the NCID-1 structure is more relaxed than that of CID-1, and this results in an increase of 
dynamics that induced the smooth diffusion of guest molecules into its 0-D pores.

In this study, we investigated the effect of downsizing the crystal of a flexible PCP from CID-1 to NCID-1 with respect to 5 crystal structure and network mobility, which would be induced by surface tension of nanoparticles. Crystallographic studies of guest-free CID-1 and NCID-1 indicated clear differences between their cell parameters, especially in the direction of the 2-D layer stacking. The changes in the two axes allow sliding of 2-D layers 10 through the flexibility of van der Waals interactions between them. These structural changes result in changes in the rotational dynamics of the organic ligand, bpy in CID-1 and NCID-1, which were confirmed by solid-state ${ }^{2} \mathrm{H}$ NMR.

The large differences in the crystal structure and molecular 15 dynamics in the bulk and nanocrystals have so far been observed, not in rigid PCP frameworks, but in flexible frameworks. NCID-1 showed downsizing effects on crystal structure, though NCID$1 \supset \mathrm{DMF}$ did not. NCID-1 $\supset \mathrm{DMF}$ with a closed pack structure undergoes a similar stable framework to that of CID-1つDMF. On 20 the other hand, nanocrystals of the guest-free NCID-1 have a structural degree of freedom because of the porous structure and large surface tension compared with CID-1, resulting the distortion of crystal structure. The results suggest that a downsizing approach contributes to the control of gas adsorption 25 with regard to the crystal structure and framework dynamics and is another strategy for control of adsorption properties such as separation and storage.

The authors thank Prof. S. Matsubara in Kyoto University for his help with the synthesis of deuterated bpy.

\section{${ }_{30}$ Notes and references}

1. (a)M. C. Daniel and D. Astruc, Chem. Rev., 2004, 104, 293; (b)H. S Nalwa, Handbook of nanostructured materials and nanotechnology, Academic Press, New York, 2000; (c)Y. N. Xia, P. D. Yang, Y. G. Sun, Y. Y. Wu, B. Mayers, B. Gates, Y. D. Yin, F. Kim and Y. Q.

35 Yan, Adv. Mater., 2003, 15, 353.

2. (a)T. J. Barton, L. M. Bull, W. G. Klemperer, D. A. Loy, B. McEnaney, M. Misono, P. A. Monson, G. Pez, G. W. Scherer, J. C. Vartuli and O. M. Yaghi, Chem. Mater., 1999, 11, 2633; (b)M. E. Davis, Nature, 2002, 417, 813; (c)M. A. Snyder and M. Tsapatsis,

40 Angew. Chem. Int. Ed., 2007, 46, 7560.

3. (a)M. Eddaoudi, J. Kim, N. Rosi, D. Vodak, J. Wachter, M. O'Keeffe and O. M. Yaghi, Science, 2002, 295, 469; (b)H. Chung, P. M. Barron, R. W. Novotny, H. T. Son, C. Hu and W. Choe, Cryst. Growth Des., 2009, 9, 3327; (c)O. K. Farha and J. T. Hupp, Acc. Chem. Res., 2010, 43, 1166.

4. (a)S. Kitagawa, R. Kitaura and S. Noro, Angew. Chem. Int. Ed., 2004 43, 2334; (b)G. Ferey and C. Serre, Chem. Soc. Rev., 2009, 38, 1380.

5. (a)R. Kitaura, K. Seki, G. Akiyama and S. Kitagawa, Angew. Chem. Int. Ed., 2003, 42, 428; (b)E. Barea, J. A. R. Navarro, J. M. Salas, N. Masciocchi, S. Galli and A. Sironi, J. Am. Chem. Soc., 2004, 126, 3014; (c)A. Kondo, H. Noguchi, S. Ohnishi, H. Kajiro, A. Tohdoh, Y. Hattori, W. C. Xu, H. Tanaka, H. Kanoh and K. Kaneko, Nano Lett., 2006, 6, 2581; (d)S. Horike, S. Shimomura and S. Kitagawa, Nat. Chem., 2009, 1, 695.

55 6. (a)T. Uemura and S. Kitagawa, J. Am. Chem. Soc., 2003, 125, 7814 (b)L. M. Huang, H. T. Wang, J. X. Chen, Z. B. Wang, J. Y. Sun, D. Y. Zhao and Y. S. Yan, Microporous Mesoporous Mater., 2003, 58, 105; (c)S. H. Jhung, J. H. Lee, J. W. Yoon, C. Serre, G. Ferey and J. S. Chang, Adv. Mater., 2007, 19, 121; (d)E. Biemmi, C. Scherb and T. Bein, J. Am. Chem. Soc., 2007, 129, 8054; (e)S. Hermes, T. Witte, T. Hikov, D. Zacher, S. Bahnmuller, G. Langstein, K. Huber and R. A. Fischer, J. Am. Chem. Soc., 2007, 129, 5324; (f)I. Boldog, A. B. Gaspar, V. Martinez, P. Pardo-Ibanez, V. Ksenofontov, A
Bhattacharjee, P. Gutlich and J. A. Real, Angew. Chem. Int. Ed., 2008, 47, 6433; (g)L. G. Qiu, Z. Q. Li, Y. Wu, W. Wang, T. Xu and X. Jiang, Chem. Commun., 2008, 3642; (h)Z. Q. Li, L. G. Qiu, T. Xu, Y. Wu, W. Wang, Z. Y. Wu and X. Jiang, Mater. Lett., 2009, 63, 78; (i)T. Tsuruoka, S. Furukawa, Y. Takashima, K. Yoshida, S. Isoda and S. Kitagawa, Angew. Chem. Int. Ed., 2009, 48, 4739; (j)J. Cravillon, S. Munzer, S. J. Lohmeier, A. Feldhoff, K. Huber and M. Wiebcke, Chem. Mater., 2009, 21, 1410; (k)S. Diring, S. Furukawa, Y. Takashima, T. Tsuruoka and S. Kitagawa, Chem. Mater., 2010, 22, 4531; (1)M. Ma, D. Zacher, X. Zhang, R. A. Fischer and N. MetzlerNolte, Cryst. Growth Des., 2010, 11, 185; (m)P. Horcajada, T. 75 Chalati, C. Serre, B. Gillet, C. Sebrie, T. Baati, J. F. Eubank, D. Heurtaux, P. Clayette, C. Kreuz, J. S. Chang, Y. K. Hwang, V. Marsaud, P. N. Bories, L. Cynober, S. Gil, G. Ferey, P. Couvreur and R. Gref, Nat. Mater., 2010, 9, 172.

7. D. Tanaka, A. Henke, K. Albrecht, M. Moeller, K. Nakagawa, S. Kitagawa and J. Groll, Nat. Chem., 2010, 2, 410.

8. S. Horike, D. Tanaka, K. Nakagawa and S. Kitagawa, Chem. Commun., 2007, 3395

9. (a)S. M. Cohen, Chem Sci, 2010, 1, 32-36; (b)T. Gadzikwa, G. Lu, C. L. Stern, S. R. Wilson, J. T. Hupp and S. T. Nguyen, Chem. Commun., 2008, 5493

10. E. J. Cussen, J. B. Claridge, M. J. Rosseinsky and C. J. Kepert, J. Am. Chem. Soc., 2002, 124, 9574.

11. (a)J. S. Vermaak and Kuhlmannw.D, J. Phys. Chem-Us, 1968, 72 , 4150; (b)Wasserma.Hj and J. S. Vermaak, Surf. Sci., 1970, 22, 164; (c)S. Tsunekawa, K. Ishikawa, Z. Q. Li, Y. Kawazoe and A. Kasuya, Phys. Rev. Lett., 2000, 85, 3440; (d)J. Y. Zhang, X. Y. Wang, M. Xiao, L. Qu and X. Peng, Appl. Phys. Lett., 2002, 81, 2076; (e)C. Q. Sun, Prog. Solid. State Chem.., 2007, 35, 1; (f)M. I. Ahmad and S. S. Bhattacharya, Appl. Phys. Lett., 2009, 95, 191906.

95 12. V. Martinez, I. Boldog, A. B. Gaspar, V. Ksenofontov, A. Bhattacharjee, P. Gutlich and J. A. Real, Chem. Mater., 2010, 22 4271.

13. (a)S. Horike, R. Matsuda, D. Tanaka, S. Matsubara, M. Mizuno, K. Endo and S. Kitagawa, Angew. Chem. Int. Ed., 2006, 45, 7226; (b)S. Amirjalayer, M. Tafipolsky and R. Schmid, Angew. Chem. Int. Ed., 2007, 46, 463; (c)S. Takamizawa, E. Nataka, T. Akatsuka, R. Miyake, Y. Kakizaki, H. Takeuchi, G. Maruta and S. Takeda, J. Am. Chem. Soc., 2010, 132, 3783.

14. M. Yamamoto, K. Oshima and S. Matsubara, Heterocycles, 2006, 67, 353.

15. T. Araya, A. Niwa, M. Mizuno and K. Endo, Chem. Phys., 2008, 344, 291.

16. R. Tycko, Nuclear Magnetic Resonance Probes of Molecular Dynamics, 1994.

110

${ }^{a}$ Department of Synthetic Chemistry and Biological Chemistry, Graduate School of Engineering, Kyoto University, Katsura, Nishikyo-ku, Kyoto 615-8510, Japan. Fax: +81 75-383-2732; Tel: +81 75-383-2733; E-mail: kitagawa@icems.kyoto-u.ac.jp

${ }_{115}{ }^{b}$ DWI e.V. and Institute of Technical and Macromolecular Chemistry, RWTH Aachen University, Pauwelsstrasse 8, 52056 Aachen, Germany.

${ }^{c}$ Department of Chemistry, Graduate School of Natural Science \& Technology, Kanazawa University, Kakuma-machi, Kanazawa 920-1192, Japan.

$120{ }^{d}$ Japan Synchrotron Radiation Research Institute, 1-1-1, Kouto, Sayo-cho, Sayo-gun, Hyogo 679-5198, Japan.

e RIKEN, SPring-8 Center, 1-1-1, Kouto, Sayo-cho, Sayo-gun, Hyogo 679-5148, Japan.

$f$ Institute for Integrated Cell-Material Sciences (iCeMS), Kyoto 125 University, Yoshida, Sakyo-ku, Kyoto 606-8501, Japan.

g ERATO Kitagawa Integrated Pores Project, Japan Science and Technology Agency (JST), Kyoto Research Park, Bulding\#3, Shimogyo-ku, Kyoto, 600-8815, Japan.

$\dagger$ Electronic Supplementary Information (ESI) available: Synthesis of 130 materials, FE-SEM image, TGA, XRPD, IR, cell parameters, and simulated details of solid-state ${ }^{2} \mathrm{H}$ NMR spectra. CCDC reference number is 812143 (CID-1). For ESI and crystallographic data in CIF see DOI: $10.1039 / \mathrm{b} 000000 \mathrm{x} /$ 